\title{
Inability to empathize following traumatic brain injury
}

\author{
RODGER LL. WOOD AND CLAIRE WILLIAMS \\ Brain Injury Research Group, Department of Psychology, School of Human Sciences, Swansea University, Swansea, Wales
}

(Received June 15, 2007; Final Revision November 2, 2007; Accepted November 2, 2007)

\begin{abstract}
This study examines: (a) the impact of traumatic brain injury (TBI) on emotional empathy, (b) the relationship between emotional empathy and neuropsychological ability, and (c) the influence of low emotional empathy on measures of affect. Eighty-nine patients completed the Balanced Emotional Empathy Scale (BEES), a number of neuropsychological tests, some of which were ecologically valid tests of executive ability, plus two measures of affect, the Beck Anxiety Inventory (BAI) and Beck Depression Inventory (BDI). The TBI cohort showed a high frequency $(60.7 \%)$ of low emotional empathy scores compared to the control group (31\%). There was no relationship between injury severity and the ability to empathize, or between emotional empathy and neuropsychological performance. There was no evidence to suggest that low scores on affective measures influenced emotional empathy scores. A high proportion of TBI patients lack the ability to empathize, but the deficit does not appear related to any specific cognitive impairment and cannot be predicted by measures of affect. (JINS, 2008, 14, 289-296.)
\end{abstract}

Keywords: Empathy, Emotion, TBI, Affective Disorder, Neuropsychological Tests, Cognitive Flexibility.

\section{INTRODUCTION}

Empathy has been described as the "binding force" of social cognition, allowing individuals to share experiences and understand each others' perspective (Eslinger et al., 2002). However, the way "empathy" is construed can have different implications for understanding its role in social cognition. It can mean feeling what another person is feeling (emotional empathy); knowing what another person is feeling (cognitive empathy); or responding compassionately to another person's distress (compassionate empathy) (BaronCohen, 1995; Davis, 1980).

Neuropsychological studies have tended to focus on cognitive empathy. Cicerone et al. (1983) found that the ability to respond empathically depended upon cognitive flexibility. Grattan et al. (1994) compared 40 subjects with circumscribed frontal and non-frontal lesions on a cognitively based self report measure of empathy, plus three measures of cognitive flexibility (Wisconsin Card Sorting Test, Verbal Fluency, and the Alternative Uses Task). Non-frontal subjects were impaired on both cognitive flexibility and empathy but

Correspondence and reprint requests to: Professor Rodger L1. Wood, Department of Psychology, School of Human Sciences, University of Wales Swansea, Singleton Park, Swansea, SA2 8PP, UK. E-mail: r.1.wood@swansea.ac.uk in the frontal group, only dorsolateral lesions had an effect on both cognitive flexibility and cognitive empathy; orbital lesions only influenced cognitive empathy whereas medial lesions only affected cognitive flexibility. Shamay-Tsoory et al. (2004a) report similar results when they compared frontal and non-frontal patients on tests of cognitive flexibility and theory of mind, plus a cognitive empathy scale. The greatest loss of empathy was recorded in patients with right hemisphere ventromedial lesions but they failed to find a relationship between cognitive empathy and cognitive flexibility. Patients with anterior lesions were more impaired on measures of empathy than those with posterior lesions. In the posterior group, only those with right hemisphere lesions showed reduced ability to empathize.

There has been little attempt to examine the relationship between prefrontal function and emotional empathy. Shamay-Tsoory et al. (2004b) used tests of cognitive flexibility and emotional processing to examine cognitive and emotional empathy in patients with localized prefrontal lesions compared to those with parietal lesions and healthy controls. They found that orbital and medial prefrontal lesions were associated with impairment of both cognitive and emotional empathy, irrespective of lesion laterality. However, patients with right parietal lesions also exhibited similar impairment to prefrontal patients, relative to healthy con- 
trols. Therefore, whereas empathy involves an interaction between those emotional processes and cognitive functions involved in social awareness and judgment, there does not appear to be a simple or direct relationship between prefrontal function and empathy, implying that a complex network of systems and structures underpins emotional processes that rely on higher-level cognitions (ShamayTsoory et al., 2004a).

There is reason to believe that reduction in empathy may be a frequent legacy of traumatic brain injury (TBI). Such injuries often implicate prefrontal regions and their connections to limbic and posterior regions, potentially affecting the complex interaction between higher cognitive processes and emotional perception. At a clinical level, weaknesses of cognitive and/or emotional empathy may underpin many of the neurobehavioral disorders associated with TBI (Wood, 2001). Whereas it is not always easy to distinguish different types of empathy deficit at a clinical level, diminished cognitive empathy seems to be reflected in a lack of tact and social discretion, as well as poor awareness of the emotional needs and sensitivities of others. Diminished emotional empathy may be reflected by an egocentric, selfcentered attitude, which is insensitive to, or neglectful of, the needs of others, even partners and children with whom, pre-accident, they had a warm, loving relationship. Selfawareness of such states seems variable. Some braininjured people appear to lack awareness of changes in their emotional character. Others acknowledge their altered emotional state yet express indifference, in respect of its impact on loved ones. The quality of relationships can suffer when emotionally indifferent behavior and failure to offer warmth and affection stands in marked contrast to an individuals pre-accident personality This may result in relationship failure (Oddy, 2003; Wood et al., 2005), which in turn leads to increased social isolation (Endberg \& Teasdale, 2004). Considering the impact of behavior and personality changes after head trauma, relatively little research has been carried out on those emotional components that underpin many of psychosocial functioning.

There have been few attempts to examine the capacity for empathy in patients who have suffered TBI. When empathy has been addressed it has mainly involved small samples and measured in the form of cognitive empathy (Grattan \& Eslinger, 1989; Milders et al., 2003). Grattan \& Eslinger, in a study containing 40 stroke and 10 TBI cases, found that $58 \%$ of patients reported low empathy, but data for the two groups were not reported separately. Milders et al. failed to find impairment in empathy when they examined 17 TBI cases. Their sample was four years post injury (compared to Grattan \& Eslinger's samples who were one year post), leading to a suggestion that problems of empathy may improve with the passage of time. However, this theory was not supported by data from Wells et al. (2005). In a much larger study of 72 TBI cases with a mean time since injury of nine years, lack of empathy was not only present but had an adverse impact on ratings of life satisfaction made by those caring for survivors of TBI.
The present study used the Balanced Emotional Empathy Scale (BEES) (Mehrabian, 2000a), to investigate the frequency of low emotional empathy in a large sample of TBI patients, and its relationship to injury severity. Cognitive abilities were also examined to see if performance on measures of neuropsychological ability, which included tests demanding some degree of cognitive flexibility, and ecologically valid tests of executive ability, were related to emotional empathy. We also considered the relationship between the ability to empathize and measures of anxiety and depression, on the assumption that low emotional empathy could be associated with emotional blunting, potentially protecting individuals from post traumatic affective disorders.

We hypothesized that: (1) the proportion of TBI cases reporting reduced emotional empathy would exceed that in a healthy control group that were similar in age, gender, intelligence, and socio-economic status; (2) that gender bias, indicating that females have greater ability to empathize (Mehrabian, 2000a) would be amplified in a TBI cohort; (3) that relationships would not be found between an inability to empathize and performance on tests of verbal intelligence but would be found when using tests that required a greater degree of cognitive flexibility and on ecologically relevant tests of executive function; (4) consistent with other research on emotional legacies of TBI, a relationship would not be found between injury severity and the ability to empathize; (5) that inability to empathize, measured by low scores on an empathy rating scale, would correlate with low scores on the Beck Depression and Anxiety Inventories.

\section{METHOD}

\section{Participants}

A power analysis indicated that a minimum of 128 participants were required (64 in each group; medium effect size, $f=.25 ; \alpha=.05$; power $=.8 ; F(1,126)=3.9163 ; \lambda=8.00)$. Exclusion criteria for the patient group comprised a preaccident history of psychiatric and/or personality disorder; a developmental history of learning disability, based either on GP records or an estimated pre-accident IQ $<70$ (preaccident factors that could affect ability to empathize); dysphasia or any other neurological disorder that would compromise ability to complete the BEES; neuropsychological disability that threw doubt on capacity to agree to participate in the study. The age range was $22-71$ at time of assessment. Participants below the age of 22 were excluded because they could be considered socially immature (in respect of the role of the frontal lobes in social maturation), which would influence their response to the BEES. The control group was selected using the same criteria.

\section{TBI group}

The cohort consisted of 89 head injured patients that met the earlier mentioned criteria, $59(66.3 \%)$ of whom were 
male. All cases had been referred to the University Head Injury Clinic during 2004 to 2005 for advice on the management of long-term neuropsychological sequelae. The mean time between injury and assessment was 3.72 years ( $S D=3.81$ years; range $.42-17.89$ years). Injury severity was determined by the length of Post Traumatic Amnesia (PTA) (mean: 13.99 days; $S D=28.99$, range 12-10) and Glasgow Coma Scores (GCS) at the time of hospital admission (mean: 10.28; $S D=4.44$, range 3-15). Mean age at injury was 38.70 years $(S D=12.052$, range $20-69)$ and at assessment, 42.33 years $(S D=11.835$, range $22-71)$. The cohort had achieved an average of 11.72 years of education ( $S D=1.994$, range 7-17). Pre-morbid intelligence was estimated using the National Adult Reading Test (NART-2) (mean 96.60; $S D=13.474$, range 70-121). Prior to injury, $86.4 \%$ of the cohort was in full time employment. At the time of assessment, $57.5 \%$ were either unemployed or working as volunteers.

\section{Control group}

This consisted of 84 participants, of whom 51 (60.7\%) were male. The mean age of participants at assessment was 40.29 years $(S D=11.921$, range 22-62). Pre-morbid intelligence was estimated using the National Adult Reading Test (NART-2) (mean 99.60; $S D=8.869$, range 82-120). At the time of assessment $90.4 \%$ of the control group were in full time employment. None of the control cohort had a formal history of psychiatric illness or any kind of pre-injury personality disorder that could be interpreted as evidence of reduced ability to empathize. The TBI and control group were compared on standard demographic information. The two groups did not differ on gender $(t(171)=-.759, p>$ $.05)$, age at assessment $(t(170)=1.128, p>.05)$, socioeconomic status (determined by pre-accident occupational level-Freelance Interview) $\left(d f=4, \chi^{2}=5.093, p=>.05\right)$, or estimated intellectual level, measured by NART-2 scores $(t(163)=-1.69, p>.05)$.

\section{Measures}

The following sub-tests from the Wechsler Adult Intelligence Scale-3rd Edition (Wechsler, 1997), were administered as part of a standard neuropsychological examination of patients referred to the clinic. For the purpose of this study, sub-tests were grouped into domains according to whether they comprised tests of verbal ability or tests that, in the author's opinion, involved a degree of cognitive flexibility.

Verbal Ability: Vocabulary, Similarities, Comprehension

Cognitive flexibility: Block Design, Matrix Reasoning, Letter-Number Sequencing, Picture Arrangement

In addition, three relatively new tests of executive ability were included, which are reported as having good ecological validity, suggesting that test performance should reflect social and functional abilities relevant to real world settings (Burgess \& Shallice, 1997; Wilson et al., 1996).

- Zoo Map Test: a measure of planning ability from the Behavioural Assessment of Dysexecutive Syndrome (Wilson et al., 1996). Inter-rater reliability across the BADS tests range from .88-1.00.

- Hayling Test: a measure of response initiation speed and response suppression. Test-retest reliability of the overall score on the Hayling Test has been reported as 0.76 (Burgess \& Shallice, 1997).

- The Brixton Test: measures rule detection and is considered by the authors to measure very similar abilities to the Wisconsin Card Sorting Test. Test-retest coefficient of 0.71 (Burgess \& Shallice, 1997).

Tests of ecological validity are designed so that test performance predicts social and functional abilities relevant to real world settings. Chaytor \& Schmitter-Edgecombe (2003) have proposed two concepts on which ecologically valid tests rely; verisimilitude and veridicality. Verisimilitude reflects the degree to which a test resembles the cognitive demands of real life tasks. The related concept of veridicality refers to the degree to which performance on a neuropsychological test is empirically related to measures of everyday functioning. In this study we have utilized both types of tasks. The Zoo Map, from The BADS (Wilson et al., 1996) is based on the principle of verisimilitude whereas the Hayling and Brixton tests (Burgess \& Shallice, 1997) seem to adhere to the principle of veridicality (see Wood \& Liossi, 2006, 2007).

The Balanced Emotional Empathy Scale (BEES) (Mehrabian, 2000a) was used as a measure of emotional empathy. The BEES is designed to distinguish persons who experience more of the feelings of others from those who are generally less responsive to the emotional expressions and experiences of others. It contains 30 items ( 15 positive and 15 negatively worded questions) that participants endorse on a nine-point Likert-type scale, ranging from very strong agreement to very strong disagreement. A total score is computed for each subject by summing responses to all 15 of the positively worded items and by subtracting from this the sum of responses to all 15 of the negatively worded items. Mehrabian (2000a) has shown that BEES scores correlate positively with a general sense of emotional wellbeing. Internal consistency has been reported as .87 (Mehrabian, 1997a). Validity data for the Balanced Emotional Empathy Scale (BEES) was reported by Mehrabian (1997).

The BEES has not, to our knowledge, been used on people who have suffered TBI but has been used extensively in the general population (Mehrabian, 1997, 2000b), and with people who experience chronic pain (Danziger et al., 2006). Singer et al. (2004) used the BEES in a functional imaging study to assess cerebral activity of participants who watched a loved one receive a painful stimulus. BEES scores correlated with level of activation of the affective component of 
the pain matrix (e.g. anterior insula, anterior cingulate cortex).

Affect was measured using the Beck Anxiety Inventory (BAI) (Beck et al., 1988) and Beck Depression Inventory (BDI) (Beck et al., 1981). The BAI and BDI are standard clinical assessment tools frequently used to measure affect following TBI (e.g., Rowland et al., 2005; Wood \& Rutterford, 2006; Wood \& Williams, 2007). The cut-off points employed by the BAI and BDI are as follows: $-0-9=$ normal; $10-19=$ mild; $20-29=$ moderate $; 29+=$ severe.

\section{Statistical Analysis}

A test of a proportion to examine the significance of the difference between two independent samples was conducted on the BEES to investigate the frequency of individuals with low emotional empathy in the TBI cohort compared to the control group. The same test was used to investigate gender differences within the TBI sample. A series of oneway between groups multivariate analysis of variance were conducted within each of the cognitive domains. The relationship between injury severity and emotional empathy was examined via correlation analysis. Correlation, multiple regression and one-way analysis of variance techniques were conducted to investigate the relationship between emotional empathy and affect as measured by the BAI and BDI.

A missing value analysis was performed to identify any patterns within the missing data. Little's Missing Completely at Random Test (Little, 1988) was calculated on the cognitive, emotional empathy, severity and affective measures. The test showed no significant deviation from a pattern of values that are missing completely at random $\left(\chi^{2}=\right.$ 1475.566, $d f=106, p=0.073)$.

\section{Procedure}

Ethical approval for the study was obtained from the Department of Psychology, School of Human Sciences, University of Wales Swansea. All TBI patients were administered cognitive tests as part of a routine clinical neuropsychological examination. On completion they were administered the BEES and the Beck questionnaires. All control participants completed the BEES. For the purpose of statistical analysis, the TBI and control cohorts were divided into three groups based on their BEES emotional empathy scores (see Table 1).

The three TBI emotional empathy groups were then compared on age at assessment and gender. There was no difference on age (Low Emotional Empathy \& Average Emotional Empathy: $t(74)=-.242, p>.05$; Low Emotional Empathy \& High Emotional Empathy: $t(63)=.258$, $p>.05$; Average Emotional Empathy \& High Emotional Empathy: $t(33)=.368, p>.05)$, but a significant group difference was noted for gender $\left(d f=2, \chi^{2}=10.004, p<\right.$ .05; Low Emotional Empathy: 42 male, 12 female; Average Emotional Empathy: 13 male, 10 female; High Emotional Empathy: 4 male, 8 female).
Table 1. Emotional empathy groups

\begin{tabular}{|c|c|c|}
\hline Empathy group & $\begin{array}{l}\text { TBI group } \\
(N=89) \\
\mathrm{N}(\%)\end{array}$ & $\begin{array}{l}\text { Control } \\
\text { group } \\
(N=84) \\
\mathrm{N}(\%)\end{array}$ \\
\hline $\begin{array}{l}\text { Group } 1-\text { Low emotional empathy } \\
\quad(\text { BEES score } \leq-1.0)\end{array}$ & $54(60.7 \%)$ & $26(31 \%)$ \\
\hline $\begin{array}{l}\text { Group 2-Average emotional } \\
\text { empathy (BEES score }-.5-1.0 \text { ) }\end{array}$ & $23(25.8 \%)$ & $41(48.8 \%)$ \\
\hline $\begin{array}{l}\text { Group 3-High emotional empathy } \\
\quad \text { (BEES score } \geq 1.0 \text { ) }\end{array}$ & $12(13.5 \%)$ & $17(20.2 \%)$ \\
\hline
\end{tabular}

The three control emotional empathy groups were also compared on age at assessment and gender. There was no difference on age (Low Emotional Empathy \& Average Emotional Empathy: $t(65)=-1.302, p>.05$; Low Emotional Empathy \& High Emotional Empathy: $t(41)=-.200, p>$ .05; Average Emotional Empathy \& High Emotional Empathy: $t(56)=1.091, p>.05)$, but a significant group difference was noted for gender $\left(d f=2, \chi^{2}=6.858, p<.05\right.$; Low Emotional Empathy: 21 male, 5 female; Average Emotional Empathy: 20 male, 21 female; High Emotional Empathy: 10 male, 7 female).

This gender difference, seen in the TBI and control groups, is consistent with normative data provided in the BEES manual, indicating that males report lower levels of emotional empathy than females (Mehrabian, 2000a). We therefore investigated gender as a factor potentially influencing empathy when analyzing data.

\section{RESULTS}

\section{Prevalence of Low Empathy}

\section{TBI and control group}

The TBI cohort recorded a significantly higher proportion of low emotional empathy $(60.7 \%)$ than the control group (31\%). The proportion of 0.60 (54 out of 89 ) individuals with low emotional empathy in the TBI cohort is significantly higher than the control group proportion of 0.30 (26 out of 84$)(Z=3.919, p<.0001)$.

\section{Gender differences}

A comparison of the three empathy groups found a significant group difference for gender in the TBI cohort. We therefore carried out a test of proportion to examine differences in the frequency of low empathy between males (66.3\%) and females $(33.7 \%)$ in the TBI cohort. The proportion of .71 males with low emotional empathy (42 out of $59)$ is significantly higher than the female proportion of .4 (12 out of 30) $(Z=2.84, p<.002)$. In the control group, the proportion of .41 males with low emotional empathy (21 out of 51 ) is significantly higher than the female pro- 
portion of .15 ( 5 out of 33$)(Z=2.52, p<.005)$. A test of proportion was therefore performed to examine frequency of low emotional empathy between the TBI male (71.2\%) and control male (41.2\%) population. The proportion of 0.71 TBI males with low emotional empathy (42 out of 59) is significantly higher than the control male proportion of 0.41 (21 out of 51) $(Z=3.173, p<.0008)$. A similar comparison of the TBI female (40\%) and control female (15.2\%) population showed that the proportion of 0.4 TBI females with low emotional empathy (12 out of 30 ) is significantly higher than the control female population proportion of 0.15 (5 out of 33) $(Z=2.219, p<.01)$.

\section{Neuropsychological Correlates of Emotional Empathy}

\section{Cognitive domains}

Measures of central tendency for the full TBI cohort on all neuropsychological tests can be found in Table 2 .

A one-way between subjects multivariate analysis of variance was conducted within each cognitive domain. There were no significant group differences in emotional empathy scores (low, average, high) for any of the cognitive domains, therefore, Bonferroni adjusted alpha levels were not necessary: 'Verbal Ability' $N=82, \mathrm{~F}(3,77)=.442, p>.05$, Wilks' $\lambda=0.966$; "Cognitive Flexibility" $N=72, F(4,66)=$ $1.175, p>.05$, Wilks' $\lambda=0 . .871$; "Ecological Ability" $N=42, F(4,36)=0.282, p>.05$, Wilks $\lambda=0.940$. PostHoc analysis revealed no significant emotional empathy group differences on any of the sub-tests within each domain: "Vocabulary" $F(2,79)=0.442, p>.05$; "Similarities" $F(2,79)=0.518, p>.05$; "Comprehension" $F(2,79)=$ $0.320, p>.05$; "Picture arrangement" $F(2,69)=1.471$, $p>.05$; "Block Design" $F(2,69)=1.638, p>.05$; "Matrix Reasoning" $\mathrm{F}(2,69)=1.046, p>.05$; "Letter Number Sequencing" $F(2,69)=.523, p>.05$; "Hayling B" $F(2,39)=.658, p>.05$; "Hayling C" $F(2,39)=.096$, $p>.05$; "Brixton" $F(2,39)=.005, p>.05$; "Zoo Map" $F(2,39)=.190, p>.05$.

\section{Influence of Injury Severity}

\section{Severity in relation to empathy}

A Pearson Product-Moment Correlation failed to establish a significant relationship between measures of emotional empathy and severity of injury as determined by length of PTA $(r=.178, p>.05)$ or Glasgow Coma Scores (GCS) $(r=-.062, p>.05)$.

\section{Severity as a factor relating empathy to neuropsychological ability}

Relationships between emotional empathy and sub-test groups were examined again, this time including only the most seriously injured cases, determined by length of PTA ( $\geq 7$ days) (mean $P T A=32.06$ days, $S D=38.22$ ). Length
Table 2. Central tendencies-cognitive functioning

\begin{tabular}{|c|c|c|c|}
\hline Test & $N$ & Mean & $S D$ \\
\hline \multicolumn{4}{|l|}{ Vocabulary } \\
\hline Low emotional empathy & 53 & 8.57 & 2.70 \\
\hline Average emotional empathy & 21 & 7.95 & 2.41 \\
\hline High emotional empathy & 12 & 8.33 & 2.30 \\
\hline \multicolumn{4}{|l|}{ Similarities } \\
\hline Low emotional empathy & 53 & 7.92 & 2.84 \\
\hline Average emotional empathy & 21 & 7.57 & 2.61 \\
\hline High emotional empathy & 11 & 8.27 & 2.79 \\
\hline \multicolumn{4}{|l|}{ Comprehension } \\
\hline Low emotional empathy & 50 & 8.46 & 3.46 \\
\hline Average emotional empathy & 21 & 8.14 & 2.93 \\
\hline High emotional empathy & 12 & 7.92 & 2.31 \\
\hline \multicolumn{4}{|l|}{ Block design } \\
\hline Low emotional empathy & 53 & 8.96 & 2.27 \\
\hline Average emotional empathy & 22 & 9.68 & 2.29 \\
\hline High emotional empathy & 12 & 8.67 & 2.49 \\
\hline \multicolumn{4}{|l|}{ Matrix reasoning } \\
\hline Low emotional empathy & 50 & 9.96 & 3.20 \\
\hline Average emotional empathy & 20 & 9.20 & 2.52 \\
\hline High emotional empathy & 10 & 9.50 & 2.41 \\
\hline \multicolumn{4}{|l|}{ Letter number sequencing } \\
\hline Low emotional empathy & 49 & 8.20 & 3.34 \\
\hline Average emotional empathy & 18 & 7.94 & 3.78 \\
\hline High emotional empathy & 10 & 8.10 & 3.14 \\
\hline \multicolumn{4}{|l|}{ Picture Arrangement } \\
\hline Low emotional empathy & 51 & 8.65 & 1.17 \\
\hline Average emotional empathy & 21 & 8.52 & 1.40 \\
\hline High emotional empathy & 10 & 9.10 & 1.44 \\
\hline \multicolumn{4}{|l|}{ Zoo Map } \\
\hline Low emotional empathy & 39 & 2.90 & 1.11 \\
\hline Average emotional empathy & 19 & 2.74 & 1.24 \\
\hline High emotional empathy & 8 & 2.63 & 1.06 \\
\hline \multicolumn{4}{|l|}{ Hayling B } \\
\hline Low emotional empathy & 45 & 5.60 & 0.86 \\
\hline Average emotional empathy & 18 & 5.72 & 0.95 \\
\hline High emotional empathy & 8 & 6.00 & 0.00 \\
\hline \multicolumn{4}{|l|}{ Hayling C } \\
\hline Low emotional empathy & 45 & 5.67 & 2.17 \\
\hline Average emotional empathy & 18 & 5.83 & 2.17 \\
\hline High emotional empathy & 8 & 5.75 & 1.90 \\
\hline \multicolumn{4}{|l|}{ Brixton } \\
\hline Low emotional empathy & 28 & 5.96 & 1.75 \\
\hline Average emotional empathy & 16 & 5.94 & 2.12 \\
\hline High emotional empathy & 5 & 6.00 & 1.87 \\
\hline
\end{tabular}

of PTA, rather than GCS scores were used because PTA has been shown to be a better indicator of neuropsychological sequelae (e.g. Bishara et al., 1992; Stambrook et al., 1993; Wilson et al., 1993). The cohort consisted of 36 cases, of whom 4 (11.1\%) scored $\geq 1.0$ (high emotional empathy; group 3), $12(33.3 \%)$ scored $-0.5-1.0$ (average emotional empathy; group 2), and $20(55.6 \%)$ scored $\leq-1.0$ (low emotional empathy; group 1). A one-way between subjects 
multivariate analysis of variance was conducted within each cognitive domain. There were no significant group differences in emotional empathy scores (low, average, high) for any of the cognitive domains, therefore, Bonferroni adjusted alpha levels were unnecessary: "Verbal Ability" $N=34$, $\mathrm{F}(3,29)=0.538, p>.05$, Wilks' $\lambda=.897$; “Cognitive Flexibility" $N=25, F(4,19)=1.473, p>.05$, Wilks' $\lambda=$ 0.583; "Ecological Ability" $N=25, F(4,22)=.896, p>$ .05 , Wilks $\lambda=.708$. Post-Hoc analysis revealed no significant emotional empathy group differences on any of the sub-tests within each domain: "Vocabulary" $F(2,31)=0618$, $p>.05$; "Similarities" $F(2,31)=.022, p>.05$; "Comprehension" $F(2,31)=.108, p>.05$; "Picture arrangement" $F(2,22)=.880, p>.05$; "Block Design" $F(2,22)=1.220$, $p>.05$; "Matrix Reasoning" $F(2,22)=1.073, p>.05$; "Letter Number Sequencing" $F(2,22)=0.915, p>.05$; "Hayling B" $F(2,22)=.686, p>.05$; "Hayling C" $F(2,22)=$ $1.525, p>.05$; "Brixton" $F(2,22)=.243, p>.05$; "Zoo Map" $F(2,22)=0.913, p>.05$.

\section{Affective Disorder}

Although not formally diagnosed with a psychological disorder, $53.7 \%$ of group 1 (low emotional empathy), $56.7 \%$ of group 2 (average emotional empathy) and $58.3 \%$ of group 3 (high emotional empathy) reported mild or moderately low mood on the BDI (Group 1: $\mathrm{M}=19.03, S D=$ 10.21; Group 2: $M=23.31, S D=8.71$; Group 3: $M=$ $19.25, S D=9.25$ ). Additionally, $38.9 \%$ of group $1,43.5 \%$ of group 2 and $58.3 \%$ of group 3 reported mild or moderately high anxiety on the BAI (Group 1: $M=15.90, S D=$ 9.84; Group 2: $M=26.91, S D=15.64$; Group 3: $M=$ $22.25, S D=12.89$ ).

The relationship between emotional empathy and measures of mood and anxiety was measured using the Pearson Product-Moment Correlation. No significant correlation was obtained between depression and emotional empathy: $r=$ $0.083, N=55, p>.05$ or between anxiety and emotional empathy: $r=.228, N=49, p>.05$.

To investigate the possibility that emotional empathy scores may be influenced by patients who reported moderate or high levels of depression and anxiety, we used multiple regression techniques to determine if $\mathrm{BDI}$ and $\mathrm{BAI}$ scores explained the variance in emotional empathy scores. BDI and BAI scores only explained $5.7 \%$ of the variance in emotional empathy scores (adjusted R square, $p>.05$ ). Of the two model variables, neither BDI nor BAI scores made a statistically unique contribution to the prediction of emotional empathy scores (BDI: $\beta=-.091, p>.05$; BAI: $\beta=$ $.284, p>.05)$.

\section{DISCUSSION}

The results of this study suggest that many TBI patients exhibit a loss of, or reduction in, the ability to empathize. In a TBI sample, $60.7 \%$ of cases recorded low levels of emotional empathy, compared to $31 \%$ of a control group drawn from the general population. Males reported higher rates of low emotional empathy than females in TBI and control groups, consistent with gender bias for empathy. However, the TBI cohort reported significantly higher rates of low empathy for both males and females, suggesting that this important aspect of social cognition is vulnerable to head trauma, irrespective of gender. Therefore, in addition to reported emotion recognition deficits following TBI (McDonald \& Flanagan, 2004; McDonald \& Saunders, 2005) and high levels of acquired (organic) alexithymia (Wood \& Williams, 2007), TBI can also produce the kind of emotional blunting that prevents many individuals from experiencing the emotions of others, a deficit that potentially contributes to a range of interpersonal difficulties and problems of psychosocial adjustment.

There was no relationship between emotional empathy and severity of TBI, implying that even relatively minor head injury (presumably in vulnerable individuals) has the potential to alter some emotional functions important to social cognition. However, the absence of any relationship between emotional empathy and cognitive abilities, including tests of cognitive flexibility and ecologically relevant executive tests, suggests that emotional empathy, as a component of social cognition, may operate in a manner that is relatively independent of cognitive ability per se.

There was no obvious relationship between emotional empathy and measures of depression and anxiety. We failed to find a significant correlation between emotional empathy, depression, and anxiety. Furthermore, when a multiple regression technique was used to investigate the possibility that low emotional empathy scores may be a by-product of some kind of affective disturbance (as measured by the BDI and BAI), we found that measures of affect accounted for only $5.7 \%$ of the variances on the BEES. Neither depression nor anxiety made a unique contribution to emotional empathy scores, suggesting that these measures of affect should be considered distinct and independent constructs from emotional empathy.

This study has a number of limitations. The neuropsychological data is incomplete because the number and type of tests administered to patients was dependent on clinical circumstances at the time of assessment. However, a missing values analysis did not show significant deviation from a pattern of values that are missing completely at random. The grouping of tests into domains for statistical analysis represents a further limitation. The authors acknowledge that some of the tests used could have been assigned to alternative domains; however we feel there is enough clinical justification for the present grouping structures. With regard to investigating the relationship between empathy, cognitive flexibility, and executive ability, the measures used were different to measures employed in previous research (Grattan et al., 1994; Shamay-Tsoory et al., 2004a), which could have influenced the results. However, we felt that tests designed to have ecological relevance were most likely to relate to empathy. We also accept that even though the TBI cohort did not report a pre-accident history of psychi- 
atric, neurological or personality problems, potentially interpretable as a lack of empathy, sub-clinical levels may still have been present that were exacerbated by head trauma. Furthermore, based on previous research reporting high levels of acquired alexithymia following TBI (Wood \& Williams, 2007), it may be possible that what appears to be poor empathy may be the result of alexithymia. Additionally, we realize that self-report measures may be vulnerable to influence by a range of variables, including self-awareness and biased perception. We further recognize that whereas the study excluded participants below the age of 22 for reasons of potential social and cerebral immaturity, the study still included participants from the other end of the age spectrum (65-71). However, we are not aware of evidence suggesting that capacity for emotional empathy is in any way reduced in this age group. Finally, whereas the results of this study confirm that TBI can result in reduced ability to empathize, it is not possible to directly implicate prefrontal structures in a TBI group because of the diffuse nature of head trauma.

We believe that the findings of this study provide further support for the idea that neuropsychological examination should carefully assess emotional and personality changes after TBI, because disorders of emotion recognition and expression can adversely impact inter-personal relationships and the quality of psychosocial outcome. This can be especially important in cases where families report behavior and personality changes, in patients who exhibit little or no measurable cognitive impairment. Future research should examine in more detail the reliability and validity of the BEES when used with a TBI cohort in order to improve the ability to recognize, at an early stage of recovery, subtle emotional deficits that may have long-term psychosocial consequences. Future research should also examine relationships between acquired alexithymia and empathy, to see if these are distinct or overlapping constructs.

\section{ACKNOWLEDGMENTS}

The information in this manuscript and the manuscript itself is new and original and has never been published either electronically or in print. The authors have no financial relationships or conflict of interest to disclose.

\section{REFERENCES}

Baron-Cohen, S. (1995). Mind blindness: An essay on autism and theory of mind. Cambridge, MA: MIT Press.

Beck, A.T., Epstein, N., Brown, G., \& Steer, R.A. (1988). An inventory for measuring clinical anxiety: Psychometric properties. Journal of Consulting and Clinical Psychology, 56, 893-897.

Beck, A.T., Ward, C.H., Mendelson, M., Mock, J., \& Erbaugh, J. (1981). An inventory for measuring depression. Archives of General Psychiatry, 4, 561-571.

Bishara, S.N., Partridge, F.M., Godfrey, H.P.D., \& Knight, R.G. (1992). Post-traumatic amnesia and Glasgow Coma Scale related to outcome in survivors in a consecutive series of patients with severe closed-head injury. Brain Injury, 3, 373-380.
Burgess, P.W. \& Shallice, T. (1997). The Hayling and Brixton Tests. Bury St. Edmunds, England: Thames Valley Test Company Limited.

Chaytor, N. \& Schmitter-Edgecombe, M. (2003). The ecological validity of neuropsychological tests: A review of the literature on everyday cognitive skills. Neuropsychology Review, 13, 181-197.

Cicerone, K.D., Lazar, R.M., \& Shaperio, W.R. (1983). Effects of frontal lobe lesions on hypothesis sampling during concept formation. Neuropsychologia, 21, 513-524.

Danziger, N., Prkachin, K.M., \& Willer, J.C. (2006). Is pain the price of empathy? The perception of other's pain in patients with congenital insensitivity to pain. Brain, 126, 2494-2507.

Davis, M.H. (1980). A multidimensional approach to individual differences in empathy. JCAS Catalogue of Selected Documents in Psychology (MS No. 2124); 10, 1-17.

Endberg, A.W. \& Teasdale, T.W. (2004). Psychosocial outcome following traumatic brain injury in adults: A long term population based follow up. Brain Injury, 18, 533-545.

Eslinger, P.J., Parkinson, K., \& Shamay, S.G. (2002). Empathy and social-emotional factors in recovery from stroke. Current Opinion in Neurology, 15, 91-97.

Grattan, L.M., Bloomer, R.H., Archambault, F.X., \& Eslinger, P.J. (1994). Cognitive flexibility and empathy after frontal lobe lesion. Neuropsychiatry, Neuropsychology and Behavioral Neurology, 7, 251-259.

Grattan, L.M. \& Eslinger, P.J. (1989). Higher cognition and social behaviour: Changes in cognitive flexibility and empathy after cerebral lesions. Neuropsychology, 38, 175-185.

Little, R. (1988). A test of missing completely at random for multivariate data with missing values. Journal of the American Statistical Association, 83, 1198-1202.

McDonald, S. \& Flanagan, S. (2004). Social perception deficits after traumatic brain injury: Interaction between emotion recognition, mentalizing ability, and social communication. Neuropsychology, 18, 572-579.

McDonald, S. \& Saunders, J.C. (2005). Differential Impairment in recognition of emotion across different media in people with severe traumatic brain injury. Journal of the International Neuropsychological Society, 11, 392-399.

Mehrabian, A. (1997). Relations among personality scales of aggression, violence, and empathy: Validational evidence bearing on the Risk of Eruptive Violence Scale. Aggressive Behavior, 23, 433-445.

Mehrabian, A. (2000a). Manual for the Balanced Emotional Empathy Scale (BEES). Available from Albert Mehrabian, 1130 Alta Mesa Road, Monterey, CA 93040.

Mehrabian, A. (2000b). Beyond IQ: Broad-based measurement of individual success potential or "emotional intelligence." Genetic, Social, and General Psychology Monographs, 126, 133-239.

Milders, M., Fuchs, S., \& Crawford, J.R. (2003). Neuropsychological impairments and changes in emotional and social behaviour following severe traumatic brain injury. Journal of Clinical and Experimental Neuropsychology, 25, 157-172.

Oddy, M. (2003). Psychosocial consequences of brain injury. In R.J. Greenwood, M.P. Barnes, T.M. McMillan, \& C.D. Ward (Eds.), Handbook of Neurological Rehabilitation (2nd ed.). Hove, UK: Psychology Press.

Rowland, S.M., Lam, C.S., \& Leahy, B. (2005). Use of the Beck Depression Inventory-II (BDI-II) with persons with traumatic brain injury: Analysis of factorial structure. Brain Injury, 19, $77-83$. 
Shamay-Tsoory, S.G., Tomer, R., Berger, B.D., \& Aharon-Peretz, J. (2004a). Characterization of empathy deficits following prefrontal brain damage: The role of the right ventromedial prefrontal cortex. Journal of Cognitive Neuroscience, 15, 324-337.

Shamay-Tsoory, S.G., Tomer, R., Goldsher, D., Berger, B.D., \& Aharon-Peretz, J. (2004b). Impairment in cognitive and affective empathy in patients with brain lesions: Anatomical and cognitive correlates. Journal of Clinical and Experimental Neuropsychology, 26, 1113-1127.

Singer, T., Seymour, B., O’Doherty, J., Kaube, H., Dolan, J.R., \& Frith, C.D. (2004). Empathy for pain involves the affective but not sensory components of pain. Science, 303, 1157-1162.

Stambrook, M., Moore, A.D., Lubusko, A.A., Peters, L.C., \& Blumenschein, S. (1993). Alternatives to the Glasgow Coma Scale as a quality of life predictor following traumatic brain injury. Archives of Clinical Neuropsychology, 8, 95-103.

Wechsler, D. (1997). Wechsler Adult Intelligence Scale (3rd ed.). San Antonio, TX: The Psychological Corporation.

Wells, R., Dywan, J., \& Dumas, J. (2005). Life satisfaction and distress in family caregivers as related to specific behavioural changes after traumatic brain injury. Brain Injury, 19, 1105-1115.

Wilson, B.A., Alderman, N., Burgess, P.W., Emslie, H., \& Evans, J.J. (1996). Behavioural Assessment of the Dysexecutive Syndrome. Bury St. Edmunds, England: Thames Valley Test Company.
Wilson, J.T., Teasdale, G.M., Hadley, D.M., Wiedmann, K.D., \& Lang, D. (1993). Post-traumatic amnesia: Still a valuable yardstick. Journal of Neurology, Neurosurgery and Psychiatry, 56, 198-201.

Wood, R.Ll. (2001). Understanding neurobehavioural disability. In R.Ll. Wood \& T.M. McMillan (Eds.), Neurobehavioural Disability and Social Handicap following Traumatic Brain Injury (pp. 1-28). Hove, UK: Psychology Press. London: Taylor \& Francis.

Wood, R.L1. \& Liossi, C. (2006). The ecological validity of executive tests in a severely brain injured sample. Archives of Clinical Neuropsychology, 21, 429-437.

Wood, R.L1. \& Liossi, C. (2007). The relationship between general intellectual ability and performance on ecologically valid executive tests in a severe brain injury sample. Journal of the International Neuropsychological Society, 13, 90-98.

Wood, R.Ll. \& Rutterford, N.A. (2006). The impact of mild developmental learning difficulties on neuropsychological recovery from head trauma. Brain Injury, 20, 1-8.

Wood, R.L1., Liossi, C., \& Wood, L. (2005). The impact of head injury neurobehavioural sequelae on personal relationships: Preliminary findings. Brain Injury, 19, 845-851.

Wood, R.Ll. \& Williams, C. (2007). Neuropsychological correlates of alexithymia. Journal of the International Neurospychological Society, 13, 471-479. 